\title{
Hydrogen-atom excitation and ionization by proton impact in the 50- to $200-\mathrm{keV}$ energy region
}

\author{
E. Fitchard, A. L. Ford, and J. F. Reading \\ Cyclotron Institute and Department of Physics, Texas A\&M University, College Station, Texas 77843
}

(Received 28 December 1976)

\begin{abstract}
We report results for $n=2$ and $n=3$ excitation and ionization in proton-hydrogen collisions. The calculations use a single-center (target) basis with $s, p$, and $d$ angular momentum states, and include all terms in the Born series. For projectile energies between 50 and $200 \mathrm{keV}$ the results are in excellent agreement with recent experiments.
\end{abstract}

The excitation and ionization of a hydrogen atom by proton impact has been for many years, and still is, a subject of great theoretical interest. In the 30 - to $200-\mathrm{keV}$ projectile energy region being considered here there are large deviations from the first Born approximation. At the low-energy end of this region charge transfer effects are very important, and at still lower energies an expansion in $\mathrm{H}_{2}^{+}$adiabatic molecular states is the most appropriate. The most successful calculations for excitation to the $n=2$ levels in the present energy region have perhaps been close-coupling calculations in a two-centered basis. ${ }^{1}$ Calculations of this sort performed up until now, however, give a far from satisfactory treatment of excitation to higher levels, or of ionization.

We have recently reported results of $K$-shell hole production calculations, where Born terms past the first were included. ${ }^{2}$ The method used employs a single-center basis and was specifically designed for when the projectile nuclear charge is much less than that of the target atom. We feel it is of interest to apply this method to the protonhydrogen problem, and the results of such calculations form the subject of this short note.

The single-center approach substantially reduces the computational time, which in turn allows for more complete basis sets to be used for calculation of ionization and excitation to higher levels. An important earlier calculation in the single-center basis is that of Cheshire and Sullivan. ${ }^{3}$ They performed a direct numerical integration of the time-dependent Schrödinger equation for protonhydrogen collisions in the impact parameter (straight-line constant-velocity path) approximation, by expanding the wave function in terms of target angular momentum eigenstates. Retaining only target $s$ states, they calculated $1 s \rightarrow 2 s, 3 s$, etc. excitation and also ionization cross sections. With $s$ and $p$ target angular momentum states they calculated $1 s \rightarrow 2 s$ and $1 s \rightarrow 2 p$ excitation cross sections. Later in this paper we will make detailed comparison of our results to theirs. If viewed solely within the framework of the proton-hydrogen problem, the present calculations are in a sense merely an extension of the Cheshire-Sullivan work to include $d$ states for $n=2$ excitation, and $p$ and $d$ states for $n=3$ excitation and ionization.

As in our previous calculations, we use a straight-line constant-velocity path for the projectile. The projectile thus serves to provide a timedependent perturbation $V(\overrightarrow{\mathrm{r}}, t)$ on the target atom. Our previous work centered on calculating only the first and second Born amplitudes. Here, where a projectile-to-target atomic number ratio of unity gives a very much larger coupling constant, higher Born terms are possibly much more important. For this reason a time-development $U$-matrix approach, described briefly before, was used.

The $U$-matrix method consists of writing the $U$ matrix which connects the wave function at time $t_{1}$ to that of time $t_{3}$ as

$$
\begin{aligned}
\Psi_{I}\left(t_{3}\right) & =U\left(t_{3}, t_{1}\right) \Psi_{I}\left(t_{1}\right) \\
& \approx \exp \left[-\frac{i}{\hbar v} \int_{t_{1}}^{t_{3}} V_{I}\left(t^{\prime}\right) d t^{\prime}\right] \Psi_{I}\left(t_{1}\right) .
\end{aligned}
$$

The matrix $U\left(t_{3}, t_{1}\right)$ is then calculated by expanding the exponential in a power series, up to the fourth power in $V_{I}$. Here $\Psi_{I}$ is the vector representing the wave function in the interaction picture, and $V_{I}$ is the matrix of the interaction potential. To solve the scattering problem we must calculate $\Psi_{I}$ at $t=\infty$, which is done by taking a product of matrices. At $t=-\infty$ the system starts out in some specific initial state. A $U$-matrix method was first used in Zimmerman's thesis ${ }^{4}$ for the proton-hydrogen problem and has since been applied independently by Shakeshaft. ${ }^{1}$

Use of the time-reversal properties of the $U$ matrix allowed us to obtain $U(\infty,-\infty)$ from a calculation of only $U(\infty, 0)$. The unitarity of $U(\infty, 0)$ was monitored. It is satisfactory unitarity that requires that terms thru $V_{I}^{4}$ be kept in the expansion of $U\left(t_{3}, t_{1}\right)$ for low energies and small impact parameters. 
An important part of the present calculation is the choice of the basis states for which the matrices $V_{I}$ and $\Psi_{I}$ are computed. As in our earlier work, we use basis states that result from a diagonalization of the target Hamiltonian (in this case, hydrogenic) in a set of Hilbert functions, all centered on the target atom. The resulting eigenvectors constitute an accurate representation of the first few bound states, and a discrete representation of the continuum. Basis states corresponding to target angular momenta $s, p$, and $d$ were kept.

The present calculations are somewhat more expensive than those previously reported, ${ }^{2}$ where only the first and second Born terms were computed. With our largest basis (10s, 22p, and $21 d$ states) the cross-section calculation (excitation and ionization) takes approximately 7 min per energy on an Amdahl 470V/6. The timing of the method is much more favorable if applied to a multielectron target, since cross sections for all initial states are simultaneously computed. Indeed, we are now in the process of applying the method to multiple ionization of atoms.

Our calculated cross sections for $1 s \rightarrow 2 l$ and $1 s \rightarrow 3 l$ excitation are given in Figs. 1 and 2 , where comparison is made to our first Born result and to the recent experiments of Park et al. ${ }^{5}$ All the experimental data has been renormalized to our calculated $n=2$ excitation cross section at $200 \mathrm{keV}$, rather than to the first Born result, as the data was originally reported. This lowers all the experimental values by the factor 0.928 . For projectile energies greater than $50 \mathrm{keV}$ the agreement with experiment is good. The apparent failure of our calculations at lower energies presum-

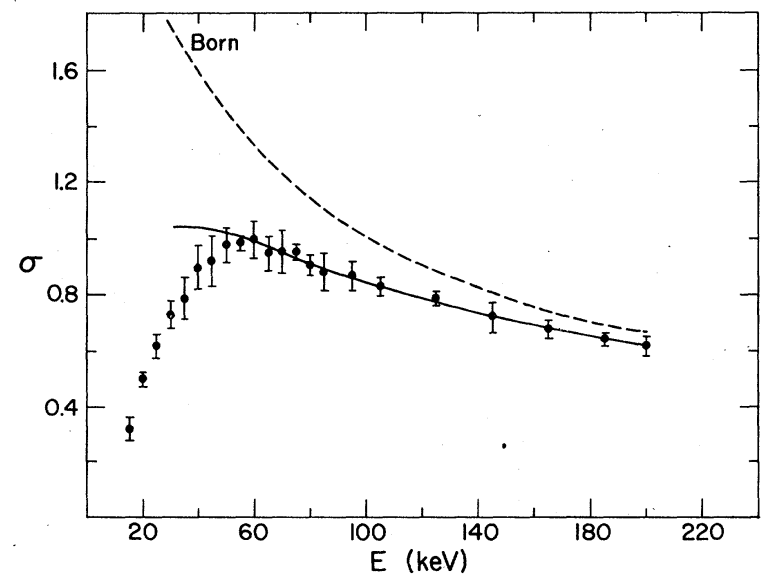

FIG. 1. Cross section (in units of $10^{-16} \mathrm{~cm}^{2}$ ) for $n=2$ excitation. The dashed curve is the first Born, the solid curve is the present calculation, and the experimental points are from Ref. 5 .

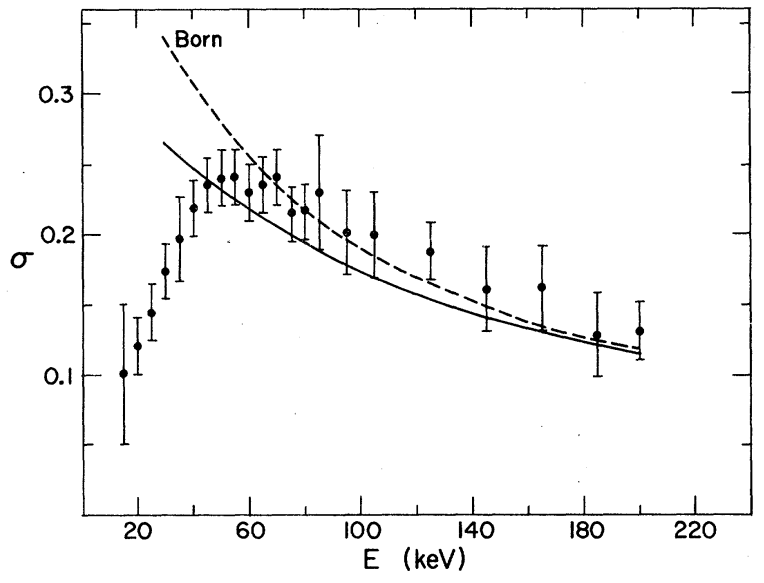

FIG. 2. Cross section (in units of $10^{-16} \mathrm{~cm}^{2}$ ) for $n=3$ excitation. The dashed curve is the first Born, the solid curve is the present calculation, and the experimental points are from Ref. 5 .

ably arises from a breakdown of the single-centered $s, p$ and $d$-state basis. It is at about $50 \mathrm{keV}$ and below that the charge exchange cross section becomes comparable to the excitation cross section. ${ }^{1}$ For $n=2$ excitation there have been many other calculations, some of which agree with experiment equally as well as ours (notably those of Shakeshaft ${ }^{1}$ and of Sullivan et al. ${ }^{6}$ ). For a complete tabulation of these and other theoretical calculations see Fig. 2 of the Park et al. paper. ${ }^{5}$ For $n$ $=3$ excitation, however, our results are superior to previous calculations (for $E>50 \mathrm{keV}$ ). Tables I and II give selected $2 s, 2 p$ and $3 s, 3 p, 3 d$ excitation cross sections from our full calculation and also in the first Born approximation. These results demonstrate that the higher-order Born terms have qualitatively different effects for different final states.

In terms of our discrete representation of the continuum, ionization cross sections are calculated in the same manner (and in the same computation) as excitation cross sections. We sum the cross sections for transitions to each of our final

TABLE I. $1 s \rightarrow 2 s, 1 s \rightarrow 2 p$ cross sections (in units of $\left.10^{-16} \mathrm{~cm}^{2}\right)$.

\begin{tabular}{ccccc}
\hline \hline & \multicolumn{2}{c}{$1 s \rightarrow 2 s$} & \multicolumn{2}{c}{$1 s \rightarrow 2 p$} \\
$E(\mathrm{keV})$ & Full calc. & First Born & Full calc. & First Born \\
\hline 30 & 0.2880 & 0.2496 & 0.7562 & 1.5105 \\
40 & 0.2242 & 0.1992 & 0.8187 & 1.4006 \\
60 & 0.1592 & 0.1414 & 0.8345 & 1.1894 \\
105 & 0.0947 & 0.0854 & 0.7426 & 0.8965 \\
200 & 0.0498 & 0.0464 & 0.5654 & 0.6164 \\
\hline \hline
\end{tabular}


TABLE II. $1 s \rightarrow 3 s, 1 s \rightarrow 3 p$, and $1 s \rightarrow 3 d$ cross sections (in units of $10^{-16} \mathrm{~cm}^{2}$ ).

\begin{tabular}{ccccccc}
\hline \hline & \multicolumn{2}{c}{$1 s \rightarrow 3 s$} & \multicolumn{2}{c}{$1 s \rightarrow 3 p$} & \multicolumn{1}{c}{$1 s \rightarrow 3 d$} \\
& Full calc. & First Born & Full calc. & First Born & Full calc. & First Born \\
\hline 30 & 0.0745 & 0.0495 & 0.1444 & 0.2654 & 0.0452 & 0.0248 \\
40 & 0.0550 & 0.0396 & 0.1478 & 0.2445 & 0.0452 & 0.0216 \\
60 & 0.0363 & 0.0282 & 0.1475 & 0.2092 & 0.0349 & 0.0168 \\
105 & 0.0206 & 0.0170 & 0.1291 & 0.1555 & 0.0197 & 0.0110 \\
200 & 0.0103 & 0.0092 & 0.0959 & 0.1048 & 0.0088 & 0.0063 \\
\hline \hline
\end{tabular}

states, excluding the final states that correspond to true bound states. If this separation of excitation from ionization transitions is to be done accurately, the basis states used must represent well the lowest few bound states. Enough of the bound states must be well represented so that those not well represented have very small excitation cross sections. Of course, to compute accurate ionization cross sections it is also necessary that the basis sets used give a good representation of the continuum. As a partial test of the adequacy of our basis set we compared first Born ionization cross sections calculated in the above described manner, with $s$ and $p$ final states only, to those computed by a partial-wave version of the exact (for hydrogen) technique of Merzbacher and Lewis. ${ }^{7}$ The agreement was excellent ( $\sim 2 \%$ difference).

Our ionization cross section results are given in Fig. 3. Our theoretical curve was determined by calculating the difference between our cross section and the first Born cross section, where $s, p$, and $d$ angular momentum states were used in each calculation. This difference was then added to the

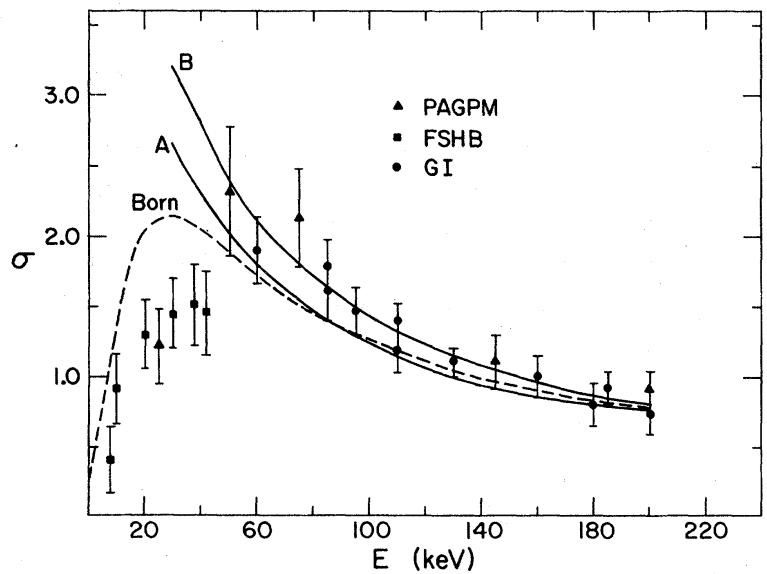

FIG. 3. Cross section (in units of $10^{-16} \mathrm{~cm}^{2}$ ) for ionization. The dashed curve is the first Born. Solid curves $\mathrm{A}$ and $\mathrm{B}$ are the present calculation where $s$ and $p$ (curve A) and $s, p$, and $d$ states (curve B) are included, respectively. The experimental points are: PAGPM, Ref. 10; FSHB, Ref. 8; and GI, Ref. 9. full first Born calculation done by the Merzbacher-Lewis method, where all angular momentum final states are included. In our $s-p-d$ first Born calculation the $d$ final states contribute about $22 \%$. This Born calculation is about $12 \%$ below the full first Born calculation. The theoretical calculation is compared to experimental results of Fite et $a l .,{ }^{8}$ Gilbody and Ireland, ${ }^{9}$ and the recently published work of Park et al. ${ }^{10}$ As was done for the excitation cross sections, the Park et al. data was renormalized down by 0.928 . Again, for $E>50 \mathrm{keV}$ our calculation is in excellent agreement with the experiment, but fails at lower energies. As for excitation, our one-center expansion must be breaking down at lower energies. In particular, our procedure is a very inefficient means for representing charge transfer type processes. To see the effect of $d$ final states, the results of including only $s$ and $p$ final states are also given.

All of the above results were obtained with a basis containing $10 s, 22 p$, and $21 d$ states. Of these, $4 s, 6 p$, and $12 d$ states represent bound states, while the rest represent the ionization continuum of the target. The $n=2$ and $n=3$ states are all essentially exactly represented. An important question is the extent to which our calculations have converged with respect to the number of $s$, $p$, and $d$ states we take. As a partial test of this we used several different basis sets at $40 \mathrm{keV}$, and found the results to be fairly insensitive to the basis being used. That we get very good first Born ionization cross sections from our discrete basis shows that in at least this sense the ionization continuum of states is well represented. As a further test of our basis, and indeed as a check of all aspects of the correctness and numerical accuracy of our calculations, we performed calculations where only $s$ and only $s$ and $p$ angular momentum states were used. These results should then be identical to the zero and first order, respectively, calculations of Cheshire and Sullivan. With only $s$ states our excitation cross sections are within $2 \%$ of those of Cheshire, over the energy range $25-200 \mathrm{keV}$. The corresponding ionization cross sections differ by $6 \%$ or less. With both $s$ 
and $p$ states the $2 s$ and $2 p$ excitation cross sections agree to within $6 \%$, except at $25 \mathrm{keV}$ where the discrepancy in the $2 p$ cross section is $13 \%$. Most of this discrepancy arises from the $1 s \rightarrow 2 p_{0}$ part of the cross section. We feel that a $6 \%$ or less error is consistent with what we would expect from our numerical methods and from our lack of basis set convergence. We do not understand the origin of the larger error for $1 s-2 p$ at $25 \mathrm{keV}$. In this regards we point out again that the use of a singlecenter basis leaves our calculation, as well as that of Cheshire and Sullivan, with little or no physical meaning for energies much below $50 \mathrm{keV}$.

Single-center basis calculations for the $n=2$ and $n=3$ excitation have also been carried out by Holt and Moiseiwitsch using their simplified second Born method. ${ }^{11}$ This method uses a single-center basis with $s, p, d$, and $f$ states. Only the first and second Born terms are evaluated, the second Born term in an approximate manner. Assuming that their simplified second Born calculation is a reasonably good approximation to the correct second Born amplitude and that $f$ states are relatively unimportant, the comparison between their calculation and ours indicates the importance of terms past the second in the Born series. Although they get substantial corrections to the Born for $s$ and for $p$ final states, these corrections are of the opposite sign and tend to cancel when $n=2$ and $n=3$ excitation is computed. Hence their $n=2$ and $n=3$ excitation results lie close to the Born.

We note that our calculations show a decrease from the first Born for $n=2$ and $n=3$ excitation, but an increase for ionization. As can be inferred from Fig. 3 this is due to two effects. First, for $d$ final states the correction to the first Born that we calculate is positive. The $d$ final states are important in ionization, as the figure shows, and are of course absent in the $n=2$ excitation and have small cross sections in the $n=3$ excitation. Second, the correction to the Born for $p$ final states, while negative at high energies in the ionization calculations, becomes positive for $E<50 \mathrm{keV}$. Tables I and II show that for excitation to $n=2$ and $n=3$ levels the correction to the first Born is, at the energies we have considered, always positive for $s$ final states and negative for $p$ final states.

In summary, we have presented results from calculations of proton-hydrogen excitation and ionization. We feel that the calculations are highly accurate, within the framework of a single-center basis with $s, p$, and $d$ angular momentum states. Our results are all in excellent agreement with experiment, for $200 \mathrm{keV}>E>50 \mathrm{keV}$. We made no attempt to extend our calculations below $30 \mathrm{keV}$, as we feel it makes no sense to do so in a calculation such as ours that does not take proper account of charge transfer. It is also true that for ionization the Born calculation follows the experimental curve reasonably well. The Born is, however, very poor for $n=2$ excitation. In the energy region in which our calculations apply, our results fit the data for. all three of the processes we have considered.

\section{ACKNOWLEDGMENTS}

We thank J. T. Park for allowing us to use results prior to publication, R. S. Shakeshaft for many helpful discussions, and S. P. Rountree for providing us with an efficient assembly language matrix multiplication routine. This work was supported by the National Science Foundation Under Grant No. GP-33992A1, and in part by the Center for Energy and Mineral Resources at Texas $\mathrm{A} \& \mathrm{M}$.
${ }^{1}$ R. S. Shakeshaft, Phys. Rev. A 14, 1626 (1976); D. F. Gallaher and L. Wilets, Phys. Rev. 169, 139 (1968); I. M. Cheshire, D. F. Gallaher, and A. J. Taylor, J. Phys. B 3, 813 (1970); D. Rapp and D. Dinwiddie, J. Chem. Phys. 57, 4919 (1972).

${ }^{2} J$. F. Reading, A. L. Ford, and E. F. Fitchard, Phys. Rev. Lett. 36, 573 (1976); Phys. Rev. A 16, 133 (1977).

${ }^{3}$ I. M. Cheshire and E. C. Sullivan, Phys. Rev. 160, 4 (1967).

${ }^{4}$ I. H. Zimmerman, thesis, University of Washington, 1972 (unpublished).

${ }^{5}$ J. T. Park, J. E. Aldag, and J. M. George, Phys. Rev. Lett. 34, 1253 (1975); J. T. Park, J. E. Aldag, J. M. George, and J. L. Peacher, Phys. Rev. A 14, 608
(1976).

${ }^{6}$ J. Sullivan, J. P. Coleman, and B. H. Bransden, J. Phys. B Proc. Phys. Soc. Lond. 5 , 2061 (1972).

${ }^{7} \mathrm{E}$. Merzbacher and H. W. Lewis, in Handbuch der Physik, edited by S. Flugge (Springer, Berlin, 1958), Vol. 24, p. 166ff.

${ }^{8}$ W. L. Fite, R. F. Stebbings, D. G. Hummer, and R. T. Brackmann, Phys. Rev. 119, 663 (1960).

${ }^{9}$ H. B. Gilbody and J. V. Ireland, Proc. R. Soc. $\underline{277}, 137$ (1963).

${ }^{10}$ J. T. Park, J. E. Aldag, J. M. George, J. L. Peacher, and J. H. McGuire, Phys. Rev. A 2 508 (1977).

${ }^{11}$ A. R. Holt and B. L. Moiseiwitsch, J. Phys. B 1,36 (1968); 2, 1253 (1969); A. R. Holt, ibid. 1202 (1969). 